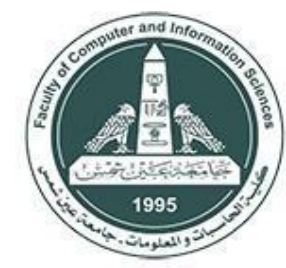

International Journal of Intelligent Computing and Information Sciences

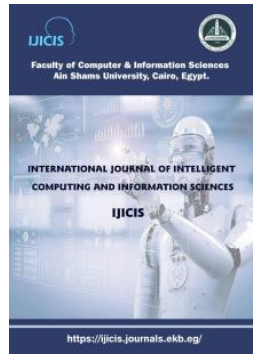

\title{
EXTRACTING RELATIONSHIPS BETWEEN THE BIG FIVE MODEL AND PERSONALITY CHARACTERISTICS IN SOCIAL NETWORKS
}

Mariam Hassanein*
Sherine Rady
Wedad Hussein
Tarek F. Gharib

Department of Information Systems, Faculty of Computer and Information Sciences, Ain Shams University, Cairo, Egypt

Mariam.hassanein@cis.asu.edu.eg $\quad$ srady@cis.asu.edu.eg Wedad.Hussein@cis.asu.edu.eg tfgharib@cis.asu.edu.eg

Received 2021- 5-21; Revised 2021-7-14; Accepted 2021-7-16

\begin{abstract}
Recently, researches focused on studying how the Big Five personality traits are manifested on social networks. These researches proved the presence of relationships between the Big Five Personality traits and various social networks features extracted from users' generated content. In this paper, the relationships between the Big Five personality traits (Openness to experience, Conscientiousness, Extraversion, Agreeableness, and Neuroticism) and attributes of personality characteristics identified as the Personal Values and Human Needs are studied. These attributes or namely features, are extracted from users' posts on social media. The relationship between the traits and proposed attributes is investigated through Pearson correlation coefficients. A dataset for 564 Twitter users is used in an experimental study, where findings proved the presence of relevant correlations between the traits and the proposed personality characteristic features. The Conscientiousness, Agreeableness, and Neuroticism traits showed strong relations existence with all of the Personal Values features, while the Openness to experience and Neuroticism traits showed strong correlations with Liberty and Selfexpression Needs features consecutively. The proposed study verified the effectiveness of the proposed Personal Values and Human Needs features as indicators for the Big Five personality traits, proving their ability for personality characteristics classification.
\end{abstract}

Keywords: Big Five, Personality Characterestics, Personal Values, Human Needs, Pearson Correlation

* Corresponding author: Mariam Hassanein

Department of Information Systems, Faculty of Computer and Information Sciences, Ain Shams University, Cairo, Egypt

E-mail address: Mariam.hassanein@cis.asu.edu.eg 


\section{Introduction}

Dramatic use of social networks like Facebook and Twitter has been witnessed over the past several years. Since their rise, people have been using them to interact with each other, build new communities, and for expressing their opinions freely [1]. Users generated content made Online social networks (OSN) a very valuable source of data that can be used for different applications. Among these applications is identifying users' personality characteristics through analyzing their digital footprints.

Based on the Five-Factor Model [2], a Human personality can be assessed in a multitude of a continuous spectrum of traits. The traits of the five-factor model are known to be desirable qualities, and often expressed as the "Big Five". The five traits form the abbreviation (O.C.E.A.N)[3][4] and include Openness to experience, Conscientiousness, Extraversion, Agreeableness, and Neuroticism. Those five dimensions define how an individual behaves, thinks, interact with the surroundings, and measure a unique aspect of a human personality. Table 1 shows a summary of the most dominant characteristics for each of the Big Five personality traits [5].

Table 1 Big Five personality Traits Characteristics

\begin{tabular}{|l|l|}
\hline \multicolumn{1}{|c|}{ Personality Trait } & \multicolumn{1}{c|}{ Characteristics } \\
\hline Openness & Imaginative, Open- minded, Insightful \\
\hline Conscientiousness & Ambitious, Organized, Self-disciplined \\
\hline Extraversion & Sociable, Assertive, Outgoing \\
\hline Agreeableness & Modest, Kind, Trusting \\
\hline Neuroticism & Nervous, Emotion instability, Anxious \\
\hline
\end{tabular}

Recently, researchers focused on studying how the personality traits are manifested on social networks. Such researches proved the presence of relationships between the Big Five traits and traditional features extracted from users' generated data (i.e., text and images) [6-8]. On the same side, many psychological studies managed to examine the relationships between the personality traits and some psychological characteristics. These characteristics have proven to be good predictors for many personality traits. Significant psychological characteristics are reflected in different characteristics identified as Personal Values and Human Needs [9]. While traits are known to be the building blocks of personality that describe how a person behaves in a certain situation, Personal Values are the beliefs that motivate such behavior and Human Needs represent an individual's desires in life.

Believing that social media can serve as a lake for analyzing and predicting human and Personal characteristics, this paper aims to investigate the existence of relationships between the famous Big Five personality traits (i.e., Openness to experience, Conscientiousness, Extraversion, Agreeableness, and Neuroticism) and personality characteristics features extracted from posts on social networks. The personality characteristics chosen are identified the Personal Values and Human Needs [4][10]. Pearson correlation coefficients are adopted to measure the strength of such relationships between the Big Five traits and the proposed features. The paper is structured as follows. Related work is given in section 2, while section 3 describes the pipeline for examining the relationships between the Big Five personality 


\section{EXTRACTING RELATIONSHIPS BETWEEN THE BIG FIVE MODEL AND PERSONALITY CHARACTERISTICS IN SOCIAL NETWORKS}

traits and Personal Values and Human Needs personality characteristics. Section 4 presents experimental results, and finally, section 5 concludes the paper.

\section{Related work}

In the past decade, a growing number of researches were dedicated to studying personality assessment using data on social networks aiming to overcome the drawbacks of the traditional ways. These studies made use of all possible features which can be extracted from an individual's personal profile. To enhance the assessment's performance, researchers focused on studying the relationships between personality traits and different social networks related features [7,11-13]. Most of these studies focused on applying text analysis to extract traditional textual features such as words count, part of speech (POS) tags, and N-grams[14].

Researchers in [15][16] aimed to study to what extent are the Big Five personality traits predictable from users' text on social media. Both depended on Linguistic Inquiry and Word Count (LIWC)[17] tool for linguistic analysis. Experiments proved the presence of many dependencies between users' personalities and language use. For example, both studies proved that Conscientiousness and Agreeableness traits were negatively correlated with LIWC categories such as negative emotion, swear, and anger. Also, Neuroticism showed a positive correlation with negative emotion and sadness categories. Finally, social process category facets were found to be positively correlated with Extraversion and Agreeableness.

A comprehensive study was conducted by [18] for two aims. Firstly, to examine the correlations between various linguistic and emotional features extracted from users' text on social media and the Big Five personality traits. Secondly to test if it is possible to generalize these correlations across different social media platforms. For this purpose, three benchmark datasets from Twitter, Facebook, and YouTube were tested. For features extraction, different linguistic analysis tools among them LIWC tool were used. Results proved the presence of correlations between six LIWC features and some personality traits among all three datasets. Eventually, different relationships were detected when using different datasets proving the impossibility to generalize the correlations between the features and the personality traits.

Recently, examining the relationships between the Big Five traits and various personality characteristics such as Personal Values and Human Needs was the scope of some psychological studies. The studies [9][19] revealed nearly consistent results regarding the relationship with Personal Values. Negative correlations were observed between Conservation value and Openness to experience, and between Agreeableness and both Hedonism and self-enhancement values. Similarly, examining relationships between the Big Five traits and Human needs proved correlations with Maslow's basic needs [20]. These needs include esteem, safety and security, self-actualization, belonging, and physiological needs. Results proved a negative correlation between Neuroticism trait and all needs. Whereas the rest of the traits were positively and significantly correlated with the needs except for Openness to experience which was not significantly correlated.

As reviewed above, most of the studies focused on investigating the relationships between the Big Five personality traits and traditional textual features extracted from data on social networks. To our 
knowledge, none of the studies addressed examining the relationships with the psychological characteristics such as Personal Values and Human Needs detected, especially in social networks posts.

\section{Big Five traits' relationship to Personality characteristics}

This study aims to investigate the relationship between the Big Five personality traits and personality characteristics represented in the Personal Values and Human Needs features. Those features are extracted from users' posts on social networks (Twitter). To investigate these relationships, three steps are executed sequentially. These steps are Data Collection, Features Extraction, and Relationship Extraction and Analysis. More explanation will be provided in the following subsections.

\subsection{Data collection}

The data used is a dataset collected from Twitter. In this step, a Twitter API is used to collect users' latest tweets using their Twitter Handles (IDs). For each user, the crawled posts are concatenated into a single document for further processing.

\subsection{Features extraction}

In this module, the personality characteristics features represented in the two categories- Personal Values and Human Needs- are extracted from users' tweets. The IBM Watson's personality Insights API was used for the features extraction. This API analyzes users' text mainly for inferring individuals' personality characteristics. The extracted features (Big Five, Personal Values and Human Needs) are based on FFM, Schwartz's Personal values theory [21], and Maslow's needs [22] respectively. Table 2 shows the 5 extracted features for the Personal Values and 12 features for Human Needs that are used in this study along with their description.

Table 2 The personal charactestics categories, Personal Values and Human Needs, along with their description

\begin{tabular}{|c|c|c|c|}
\hline No. & Characteristics & Dimension & Description \\
\hline 1 & \multirow{5}{*}{$\begin{array}{l}\text { Personal } \\
\text { Values }\end{array}$} & Hedonism & Seek pleasure and sensual enjoyment. \\
\hline 2 & & Conservation & Emphasize resistance to change and self-restriction. \\
\hline 3 & & Openness to change & $\begin{array}{l}\text { Emphasize independent action, and readiness for new } \\
\text { experiences. }\end{array}$ \\
\hline 4 & & Self-enhancement & Seek personal victory. \\
\hline 5 & & Self-transcendence & $\begin{array}{l}\text { Overcome the limits of the self and show concern for the } \\
\text { interests of others. }\end{array}$ \\
\hline 6 & \multirow{7}{*}{$\begin{array}{l}\text { Human } \\
\text { Needs }\end{array}$} & Harmony & Accept others' viewpoints, and feelings. \\
\hline 7 & & Curiosity & Have a desire to discover. \\
\hline 8 & & Challenge & Desire to take and achieve tasks \\
\hline 9 & & Stability & Seek equivalence in the physical world. \\
\hline 10 & & Ideal & Desire perfection. \\
\hline 11 & & Closeness & Enjoy being connected to family and setting up a home. \\
\hline 12 & & Excitement & Feel enthusiasm and eagerness \\
\hline
\end{tabular}




\begin{tabular}{|c|c|c|}
\hline 13 & Self-expression & Enjoy discovering and asserting own identity. \\
\hline 14 & Love & Enjoy social contact. \\
\hline 15 & Practicality & Tend to achieve clearly useful objective. \\
\hline 16 & Liberty & Behave or change with no constraints. \\
\hline 17 & Structure & $\begin{array}{l}\text { Desire to hold things together and ensure well organized and } \\
\text { under control tasks. }\end{array}$ \\
\hline
\end{tabular}

\subsection{Relationship extraction and analysis}

In this module, the relationships between the Big Five personality traits and the seventeen extracted features are investigated. Pearson correlations coefficients $(r \in[-1,1])$ presented in Equation (1) are computed.

Where

$$
r=\frac{\sum\left(X_{J}-\mu_{X_{J}}\right)\left(Y_{M}-\mu_{Y_{M}}\right)}{\sqrt{\sum\left(X_{J}-\mu_{X_{J}}\right)^{2} \sum\left(Y_{M}-\mu_{Y_{M}}\right)^{2}}}
$$

- $X_{J}$ represents a specific personality trait, with $\mathrm{J} \subset\{1,2 \ldots 5\}$ representing the Big Five traits.

- $Y_{M}$ represents a specific feature, with $\mathrm{M} \subset\{1,2 \ldots 17\}$ representing 5 Personal Values in addition to 12 Human Needs.

- $\quad \mu_{X_{J}}$ and $\mu_{Y_{M}}$ represent vectors scores mean for the $J$ trait and $M$ feature respectively.

\section{Experiments and Results}

\subsection{Dataset}

To create our dataset, Twitter handles (Twitter IDs) from a labeled dataset were entered for the Data collection to crawl users' latest tweets. The dataset contained tweets for 564 Twitter users in addition to their Big Five personality traits scores. These scores are normalized to 0-1 scale for each trait.

\subsection{Experimental results}

The relationships between the Big Five, and the Personal Values and Human Needs features extracted from users' tweets are measured as per equation (1). Table 3 shows the Pearson's correlation coefficients values with their corresponding descriptive statistics (i.e., mean, standard deviation (SD)) for all features. The correlation will be judged as high, moderate, and no correlation by analyzing the correlation coefficients values. Given that the values range from -0.65 to 0.56 , the strength of a relationship is considered to be strong when greater than or equals 0.5 , while moderate when the absolute value of $r$ is between 0.25 and 0.49 . Values outside those ranges map to no correlation. It is worth mentioning that the obtained values are also similar to an experimental study conducted in [9], 
and a similar assumption like ours was mentioned. Table 3 highlights those two categories in yellow and cyan colors respectively.

Table 3 Pearson correlation coefficients and the descriptive statistics for all features

\begin{tabular}{|c|c|c|c|c|c|c|c|}
\hline \multicolumn{2}{|r|}{ Features } & Mean (SD) & $\mathbf{O}$ & $\mathbf{C}$ & $\mathbf{E}$ & $\mathbf{A}$ & $\mathbf{N}$ \\
\hline \multirow{5}{*}{$\begin{array}{c}\text { Personal } \\
\text { Values }\end{array}$} & Conservation & $0.78(0.02)$ & $-0.33 * * * *$ & $0.56 * * * *$ & $0.11 *$ & $0.49 * * * *$ & $-0.28 * * * *$ \\
\hline & Hedonism & $0.76(0.03)$ & $-0.38 * * * *$ & $-0.65 * * * *$ & $-0.15 * * *$ & $-0.33 * * * *$ & $0.27 * * * *$ \\
\hline & Openness to change & $0.74(0.03)$ & $-0.08 *$ & $-0.63 * * * *$ & $-0.15^{* * *}$ & $-0.27 * * * *$ & $0.30 * * * *$ \\
\hline & Self-enhancement & $0.72(0.01)$ & $-0.21 * * * *$ & $-0.55 * * * *$ & $-0.13 * *$ & $-0.44 * * * *$ & $0.25 * * * *$ \\
\hline & Self-transcendence & $0.69(0.02)$ & $-0.22 * * * *$ & $-0.28 * * * *$ & -0.04 & $0.35 * * *$ & $0.33 * * * *$ \\
\hline \multirow{12}{*}{$\begin{array}{l}\text { Human } \\
\text { Needs }\end{array}$} & Liberty & $0.75(0.02)$ & $-0.44 * * * *$ & $-0.20 * * * *$ & -0.06 & -0.05 & $0.15 * * *$ \\
\hline & Ideal & $0.69(0.02)$ & $-0.25 * * * *$ & $-0.31 * * * *$ & $-0.10 *$ & $-0.14 * *$ & $0.14 * * *$ \\
\hline & Love & $0.73(0.03)$ & $-0.25 * * * *$ & $-0.23 * * * *$ & $0.23 * * * *$ & $0.26 * * * *$ & $0.27 * * * *$ \\
\hline & Practicality & $0.79(0.02)$ & $-0.35 * * * *$ & $-0.09 *$ & $-0.11 * *$ & $-0.36 * * * *$ & 0.07 \\
\hline & Self-expression & $0.84(0.02)$ & $-0.13 * *$ & $-0.14 * *$ & 0.05 & $0.19 * * * *$ & $0.25 * * * *$ \\
\hline & Stability & $0.74(0.04)$ & -0.05 & $0.35 * * * *$ & $0.20 * * * *$ & $0.38 * * * *$ & $-0.16 * * *$ \\
\hline & Structure & $0.79(0.02)$ & $-0.15 * * *$ & $0.34 * * * *$ & 0.00 & -0.02 & $-0.11 * *$ \\
\hline & Challenge & $0.63(0.03)$ & $-0.36 * * * *$ & $-0.27 * * * *$ & -0.03 & $-0.18 * * * *$ & $0.15 * * *$ \\
\hline & Closeness & $0.80(0.04)$ & $-0.28 * * * *$ & 0.00 & $0.19 * * * *$ & $0.55 * * * *$ & 0.08 \\
\hline & Curiosity & $0.81(0.03)$ & $0.09 *$ & $-0.21 * * * *$ & -0.05 & $-0.09 *$ & $0.14 * * *$ \\
\hline & Excitement & $0.72(0.03)$ & $-0.38 * * * *$ & $-0.36 * * * *$ & $-0.13 * *$ & $-0.20 * * * *$ & $0.20 * * * *$ \\
\hline & Harmony & $0.84(0.01)$ & $-0.09 *$ & $0.29 * * * *$ & $0.25 * * * *$ & $0.51 * * * *$ & 0.03 \\
\hline
\end{tabular}

Note: Openness to experience (O), Conscientiousness(C), Extraversion (E), Agreeableness (A), and Neuroticism(N), Moderate correlations are in yellow, while strong correlations are in cyan,

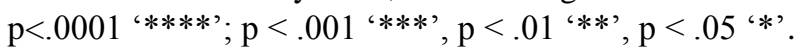

\subsubsection{Relationships between the Big Five and Personal Values}

Table 3 shows that Unlike Extraversion, correlations were observed between Conscientiousness and all Personal Values, a strong positive correlation was found with Conservation value while negative correlations were found with the rest of the values. Moreover, the Openness to experience trait is moderately and negatively correlated with Conservation and Hedonism values. The Agreeableness trait also established moderate positive correlations with Conservation and self- transcendence, while moderate negative correlations with Hedonism, Openness to change, and Self-enhancement values which agreed with results in [19]. Finally, the Neuroticism trait was moderately and positively correlated with all the values except for Conservation. In conclusion, the traits Conscientiousness, Agreeableness, and Neuroticism were proved to be significantly correlated with all Personal values. Moreover, Hedonism and Conservation features established correlation with all of the personality traits except for Extraversion. 


\section{EXTRACTING RELATIONSHIPS BETWEEN THE BIG FIVE MODEL AND PERSONALITY CHARACTERISTICS IN SOCIAL NETWORKS}

\subsubsection{Relationships between the Big Five and Human Needs}

As shown in table 3, Agreeableness trait proved the presence of strong positive correlations with Harmony and Closeness. A moderate negative correlation was proved between Agreeableness and the need for Practicality, while moderate positive correlations with the need for Love and Stability. These results agreed with the findings in [20]. Openness to experience showed the greatest number of relationships out of the five traits. This contradicts with a previous study in [20] in which the fewest number of relationships was detected with Openness to experience. Moderate negative correlations were established between Openness to experience and the need for Liberty, Ideality, Love, Practicality, Challenge, Closeness, and Excitement. Conscientiousness showed moderate negative correlations with the need for Ideality, Challenge, and Excitement. Also, moderate positive correlations were established with the need for Stability, Structure, and Harmony. Neuroticism correlated moderately and positively with the need for Love and Self-expression. Finally, with the least number of correlations, Extraversion correlated moderately and positively with the need for Harmony. In conclusion, Openness to experience was the only trait that correlated with the need for Liberty, while Neuroticism was the only trait that correlated with the need for Self-expression. Additionally, Extraversion trait correlated only with the need for Harmony.

\section{Conclusion}

This paper investigates the relationships between the Big Five personality traits and two personality characteristics features categories detected from users' posts on social networks. The two are recognized as the Personal Values and Human Needs with 5 and 12 features respectively. Pearson correlation is used to measure the relationships between the different attributes and the traits. Among the relevant observations found is that Conscientiousness, Agreeableness, and Neuroticism traits are significantly correlated with all Personal values. Additionally, all the personality traits are correlated with Hedonism and Conservation values except for Extraversion. Concerning Human needs, Openness to experience showed the highest number of relationships out of the five traits, while the Extraversion trait showed the least. Furthermore, Openness to experience was the only trait that correlated with the need for Liberty. Moreover, Neuroticism represents the only trait that correlated with the need for Selfexpression, while the Extraversion trait correlated only with the need for Harmony. In conclusion, results proved that Personal Values and Human Needs are good indicators for the Big Five personality traits which can be employed for personality traits classification.

\section{References}

1. D. Azucar, D. Marengo, M. Settanni, Predicting the Big 5 personality traits from digital footprints on social media: A meta-analysis, Personality and Individual Differences. 124 (2018) 150-159. https://doi.org/10.1016/j.paid.2017.12.018.

2. O.P. John, S. Srivastava, The Big-Five Trait Taxonomy: History, Measurement, and Theoretical Prespectives, 1999. https://doi.org/10.1109/ICARM.2016.7606898.

3. M. salim, S. Saad, M. Aref, Preprocessing the Egyptian Arabic Dialect for Personality Traits Prediction, International Journal of Intelligent Computing and Information Sciences. 19 (2019) 1-12. https://doi.org/10.21608/ijicis.2019.62603.

4. M. Hassanein, W. Hussein, S. Rady, T.F. Gharib, Predicting Personality Traits from Social Media using Text Semantics, Proceedings - 2018 13th International Conference on Computer 
Engineering and Systems, ICCES 2018, IEEE. (2019) 184-189. https://doi.org/10.1109/ICCES.2018.8639408.

5. O.P. John, O.P. John, Big Five Model of Personality, in: Encyclopedia of Quality of Life and Well-Being Research, 2014: pp. 394-394. https://doi.org/10.1007/978-94-007-0753-5_100285.

6. S. Han, H. Huang, Y. Tang, Knowledge of words: An interpretable approach for personality recognition from social media, Knowledge-Based Systems. 194 (2020) 105550. https://doi.org/10.1016/j.knosys.2020.105550.

7. L. Burdick, R. Mihalcea, R.L. Boyd, J.W. Pennebaker, Analyzing Connections Between User Attributes, Images, and Text, Cognitive Computation. (2020). https://doi.org/10.1007/s12559019-09695-3.

8. F. Mairesse, M.A. Walker, M.R. Mehl, R.K. Moore, Using linguistic cues for the automatic recognition of personality in conversation and text, Journal of Artificial Intelligence Research. 30 (2007) 457-500. https://doi.org/10.1613/jair.2349.

9. L. Parks-Leduc, G. Feldman, A. Bardi, Personality Traits and Personal Values: A Meta-Analysis, Personality and Social Psychology Review. 19 (2015) 3-29. https://doi.org/10.1177/1088868314538548.

10. S.H. Schwartz, An Overview of the Schwartz Theory of Basic Values, Online Readings in Psychology and Culture. 2 (2012) 1-20. https://doi.org/10.9707/2307-0919.1116.

11. T. Bowden-Green, J. Hinds, A. Joinson, How is extraversion related to social media use? A literature review, Personality and Individual Differences. 164 (2020) 110040. https://doi.org/10.1016/j.paid.2020.110040.

12. Y. Bachrach, M. Kosinski, T. Graepel, P. Kohli, D. Stillwell, Personality and patterns of Facebook usage, Proceedings of the 3rd Annual ACM Web Science Conference on - WebSci '12. (2012) 24-32. https://doi.org/10.1145/2380718.2380722.

13. B. Ferwerda, M. Schedl, Predicting Personality Traits with Instagram Pictures, RecSys EMPIRE 2015: 3rd Workshop on Emotions and Personality in Personalized Systems 2015. (2015) 7-10. https://doi.org/10.1145/2809643.2809644.

14. Y. Wang, Understanding Personality through Social Media, (2015).

15. H.A. Schwartz, J.C. Eichstaedt, M.L. Kern, L. Dziurzynski, S.M. Ramones, M. Agrawal, A. Shah, M. Kosinski, D. Stillwell, M.E.P. Seligman, L.H. Ungar, Personality, Gender, and Age in the Language of Social Media: The Open-Vocabulary Approach, PLoS ONE. 8 (2013). https://doi.org/10.1371/journal.pone.0073791.

16. J. Golbeck, C. Robles, M. Edmondson, K. Turner, Predicting personality from twitter, Proceedings - 2011 IEEE International Conference on Privacy, Security, Risk and Trust and IEEE International Conference on Social Computing, PASSAT/SocialCom 2011. (2011) 149156. https://doi.org/10.1109/PASSAT/SocialCom.2011.33.

17. J.W. Pennebaker, J.W. Pennebaker, M.E. Francis, R.J. Booth, Linguistic Inquiry and Word Count, (2016).

18. G. Farnadi, G. Sitaraman, S. Sushmita, F. Celli, M. Kosinski, D. Stillwell, S. Davalos, M.F. 
Moens, M. De Cock, Computational personality recognition in social media, User Modeling and User-Adapted Interaction. 26 (2016) 109-142. https://doi.org/10.1007/s11257-016-9171-0.

19. S. Roccas, L. Sagiv, S.H. Schwartz, A. Knafo, The Big Five personality factors and personal values, Personality and Social Psychology Bulletin. 28 (2002) 789-801.

https://doi.org/10.1177/0146167202289008.

20. C. Montag, C. Sindermann, D. Lester, K.L. Davis, Linking individual differences in satisfaction with each of Maslow's needs to the Big Five personality traits and Panksepp's primary emotional systems, Heliyon. 6 (2020). https://doi.org/10.1016/j.heliyon.2020.e04325.

21. S.H. Schwartz, Universals in the content and structure of values: Theoretical advances and empirical tests in 20 countries, Advances in Experimental Social Psychology. 25 (1992) 1-65. https://doi.org/10.1016/S0065-2601(08)60281-6.

22. A.H. Maslow, A Theory of human motivation, Psychological Review. July (1943) 370-396.

\section{Web Reference}

IBM (2018). Personality insights - API reference | IBM Watson Developer Cloud. Retrieved from https://www.ibm.com/watson/developercloud/Personality-insights/api/v3/ curl.htm (accessed January 2020) 